\title{
Left ventricular diastolic dysfunction index based on non-invasive measurements
}

\author{
Chun G Schiros', Thomas S Denney², Inmaculada Aban¹, Himanshu Gupta ${ }^{1 *}$ \\ From 18th Annual SCMR Scientific Sessions \\ Nice, France. 4-7 February 2015
}

\section{Background}

Echocardiographic tissue Doppler or magnetic resonance imaging (MRI) measurements of early diastolic mitral annular velocity with other appropriate parameters are frequently used as a non-invasive diagnostic tool of diastolic dysfunction. Previously published global approach utilizing normalized left ventricular (LV) torsion shear angle volume loop ( $\phi \_$hat $V \_$hat loop) was proposed to provide a new global description of LV diastolic function. The purpose of this study was to evaluate the discriminant power of these non-invasive parameters in identifying elevated LV end-diastolic pressure (LVEDP) (i.e. LVEDP $\geq 15 \mathrm{ml}$ ) and provide a non-invasive index to predict elevated LVEDP.

\section{Methods}

A group of 23 patients with LV ejection fraction $\geq 50 \%$ without acute infarct undergoing cardiac catheterization that did not undergo percutaneous coronary intervention were studied using high-fidelity pressure measurement. Echocardiogram with flow and tissue Doppler quantification was performed on the same date of cardiac catheterization for all participants. Cine and tagged cardiac MRI were performed on all subjects, followed by comprehensive volumetric and strain analysis. The database consisted of five parameters collected from all subjects: torsion hysteresis area (THA), peak $-\mathrm{d} \phi_{-} h a t / \mathrm{d} V_{-} h a t$ at early diastole, MRI derived E/ $\mathrm{A}_{\mathrm{MRI}}$, echocardiographic derived $\mathrm{E} / \mathrm{A}$ and E/e'. Stepwise variable selection was applied to select parameters with significance level of leave out and stay in equal to 0.15 . A logistic regression classifier was used to construct the non-invasive index for identifying elevated LVEDP based on the selected parameters. The classifier's prediction performance was analyzed using a Receiver-Operating Characteristic (ROC) curve and expressed as its sensitivity, specificity, accuracy and area under the curve (AUC).

\section{Results}

Among all parameters, peak $-\mathrm{d} \phi_{-} h a t / \mathrm{d} V_{-}$hat at early diastole, has the highest chi-square score of $2.45(\mathrm{p}=0.12)$, indicating it as the best discriminator compared with others. All other variables had chi-square score $<1$ and $p$ value $>0.4$. Stepwise selection chose peak peak $-\mathrm{d} \phi \_$hat/ $\mathrm{d} V \_$hat at early diastole, at early diastole, THA and echo measured E/A to construct the logistic regression model (Table 1, Figure 1). The model predicted elevated LVEDP with sensitivity of $93 \%$, specificity of $89 \%$, accuracy of $91 \%$, and AUC of 0.94 (Figure 1).

Table 1 Summary of stepwise selection

\begin{tabular}{|c|c|c|c|c|c|c|c|}
\hline \multirow[t]{2}{*}{ Step } & \multicolumn{2}{|l|}{ Variable } & \multirow[t]{2}{*}{ Degrees of Freedom } & \multirow[t]{2}{*}{ Number In } & \multirow[t]{2}{*}{ Chi-Square Score } & \multirow[t]{2}{*}{ Wald Chi-Square } & \multirow[t]{2}{*}{$P$ value } \\
\hline & Entered & Removed & & & & & \\
\hline 1 & Peak -d $\phi$ _hat/dV_hat & & 1 & 1 & 2.45 & & 0.1174 \\
\hline 2 & Torsion Hysteresis Area & & 1 & 2 & 5.32 & & 0.0211 \\
\hline 3 & $E / A$ & & 1 & 3 & 5.08 & & 0.0242 \\
\hline 4 & Mean E/e' & & 1 & 4 & 2.66 & & 0.1031 \\
\hline 5 & & Mean E/e' & 1 & 3 & & 1.29 & 0.2552 \\
\hline
\end{tabular}

${ }^{1}$ University of Alabama at Birmingham, Birmingham, AL, USA 


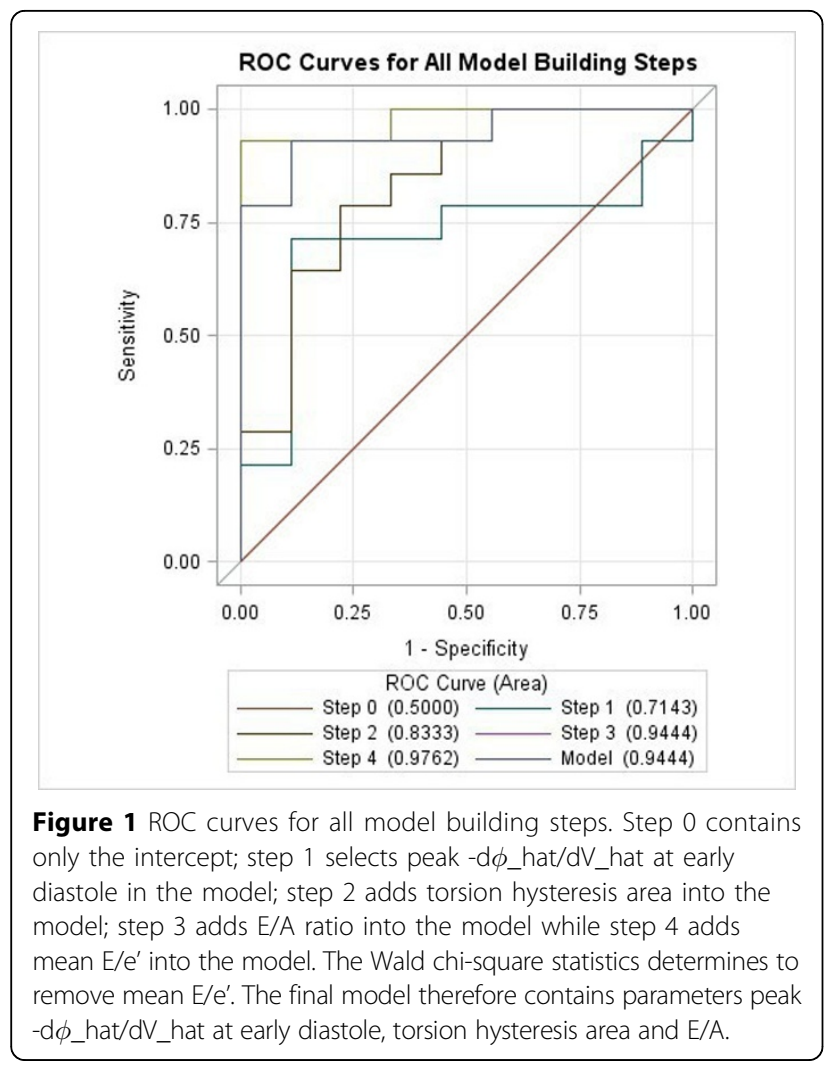

\section{Conclusions}

MRI derived global parameter peak - $\mathrm{d} \phi \phi_{-}$hat $/ \mathrm{d} V \_$hat at early diastole was the best discriminator compared with other non-invasive measures in identifying elevated LVEDP. Moreover, by combining THA, peak - d $\phi_{-}$hat/ $\mathrm{d} V \_h a t$ at early diastole and echo measured E/A through stepwise selection, the logistic regression model can identify LVEDP $\geq 15 \mathrm{ml}$ with great accuracy, indicating that these parameters are determined by different factors and together, they are able to predict diastolic dysfunction non-invasively.

\section{Funding}

NIH NHLBI R01-HL104018.

\section{Authors' details}

${ }^{1}$ University of Alabama at Birmingham, Birmingham, AL, USA. ${ }^{2}$ Auburn University, Auburn, AL, USA.

Published: 3 February 2015

doi:10.1186/1532-429X-17-S1-Q24

Cite this article as: Schiros et al: Left ventricular diastolic dysfunction

index based on non-invasive measurements. Journal of Cardiovascular Magnetic Resonance 2015 17(Suppl 1):Q24.

\section{Submit your next manuscript to BioMed Central} and take full advantage of:

- Convenient online submission

- Thorough peer review

- No space constraints or color figure charges

- Immediate publication on acceptance

- Inclusion in PubMed, CAS, Scopus and Google Scholar

- Research which is freely available for redistribution 